\title{
Percolating transport in superconducting nanoparticle films
}

Shawn Fostner, Amol Nande, Alex Smith, Rodrigo Martinez Gazoni, Jack Grigg, Kristiaan Temst, Margriet J. Van Bael, and Simon A. Brown

Citation: Journal of Applied Physics 122, 223905 (2017);

View online: https://doi.org/10.1063/1.5006694

View Table of Contents: http://aip.scitation.org/toc/jap/122/22

Published by the American Institute of Physics

\section{Scilight}

Sharp, quick summaries illuminating the latest physics research 


\title{
Percolating transport in superconducting nanoparticle films
}

\author{
Shawn Fostner, ${ }^{1,2}$ Amol Nande, ${ }^{1,2}$ Alex Smith, ${ }^{1,2}$ Rodrigo Martinez Gazoni, ${ }^{1,2}$ Jack Grigg, ${ }^{1,2}$ \\ Kristiaan Temst, ${ }^{3}$ Margriet J. Van Bael, ${ }^{4}$ and Simon A. Brown ${ }^{1,2, a)}$ \\ ${ }^{1}$ The MacDiarmid Institute for Advanced Materials and Nanotechnology \\ ${ }^{2}$ Department of Physics and Astronomy, University of Canterbury, Private Bag 4800, Christchurch 8140, \\ New Zealand \\ ${ }^{3}$ Instituut voor Kern-en Stralingsfysica, KU Leuven, Leuven, Belgium \\ ${ }^{4}$ Laboratory of Solid State Physics and Magnetism, KU Leuven, Leuven, Belgium
}

(Received 27 September 2017; accepted 14 November 2017; published online 13 December 2017)

\begin{abstract}
Nanostructured and disordered superconductors exhibit many exotic fundamental phenomena, and also have many possible applications. We show here that films of superconducting lead nanoparticles with a wide range of particle coverages, exhibit non-linear $V(I)$ characteristics that are consistent with percolation theory. Specifically, it is found that $V \propto\left(I-I_{c}\right)^{a}$, where $a=2.1 \pm 0.2$, independent of both temperature and particle coverage, and that the measured critical currents $\left(I_{c}\right)$ are also consistent with percolation models. For samples with low normal state resistances, this behaviour is observable only in pulsed current measurements, which suppress heating effects. We show that the present results are not explained by vortex unbinding [BerezinskiiKosterlitz-Thouless] physics, which is expected in such samples, but which gives rise to a different power law behaviour. Finally, we compare our results to previous calculations and simulations, and conclude that further theoretical developments are required to explain the high level of consistency in the measured exponents $a$. Published by AIP Publishing. https://doi.org/10.1063/1.5006694
\end{abstract}

\section{INTRODUCTION}

Percolation theory provides significant insight into the behavior of disordered superconducting systems such as granular films ${ }^{1-10}$ and artificial Josephson-Junction (JJ) arrays, ${ }^{11-13}$ and is especially relevant to the critical behavior of superconducting systems. ${ }^{14-22}$ More specifically, in twodimensional (2D) superconducting systems, percolation underpins our understanding of the superconductor-insulator transitions in both granular ${ }^{7-10}$ and thin film ${ }^{19-21,23-26}$ systems, the effects of inter-particle connectivity on system conductivity, ${ }^{6,7,27,28}$ and the nature of the superconducting-normal state transition. ${ }^{13,18,29-32}$ This understanding impacts on important technological issues; for example, an understanding of percolative transport between grains has been used to engineer an increase in the current-carrying capacity in high temperature superconductors (HTSCs). ${ }^{28,33}$

The transport properties of percolating superconductors often (see Refs. 28, 33-35 and references therein) exhibit power laws of the form

$$
V \sim\left(I-I_{c}\right)^{a}
$$

where $a \sim 2-2.5$ and $I_{c}$ is the measured critical current of the superconducting system. This power law results ${ }^{34-36}$ from successive transitions of weak links/bonds from the superconducting state to the normal state as the applied current is increased, and so it is often called the "breakdown form". 33 This is illustrated in Fig. 1 for a percolating network of particles (and discussed in more detail below). At low currents, the "backbone" of connected particles is in the

\footnotetext{
a)simon.brown@canterbury.ac.nz
}

superconducting state [Fig. 1(b)], but as the applied current is increased, the local critical current in some weak links is exceeded and they transition to the normal state. As I continues to increase, other weak link transitions take place in succession until eventually, at $I_{c}$, the last remaining wholly superconducting path across the system is eliminated and a voltage is generated [Fig. 1(c)]. As the applied current is increased further, all the weak links and more and more of the strongly linked particles transition to the normal state until eventually the entire "backbone" is resistive [Fig. 1(d)] and the normal state resistance $R_{N}$ of the system is measured.

While this basic mechanism is clear, the range of validity of Eq. (1) is not well understood, and the question of the universality of the exponent $a$ has not been resolved. ${ }^{34-36}$ Furthermore, it is often difficult ${ }^{33,38}$ to experimentally distinguish Eq. (1) from behaviour of the form

$$
V \sim I^{b}
$$

Equation (2) results from, for example, vortex flow ${ }^{39}$ or vortex/anti-vortex unbinding [the Berezinskii-KosterlitzThouless (BKT) transition]. ${ }^{40,41}$ The results of the forms of Eqs. (1) and (2) are described by two portions of the literature that are almost entirely disjoint: while it has long been acknowledged that both the behaviours can make a contribution to the transition between the superconducting and normal states, there have been very few attempts ${ }^{33}$ to compare the two possible interpretations of any given experimental data set. It is clear, however, that in principle, both behaviours could be observed in the same samples (in different current ranges) and that the effects of the inhomogeneity/disorder $^{13,30,42-44}$ and system size ${ }^{31,45,46}$ will further complicate matters. 
(a)

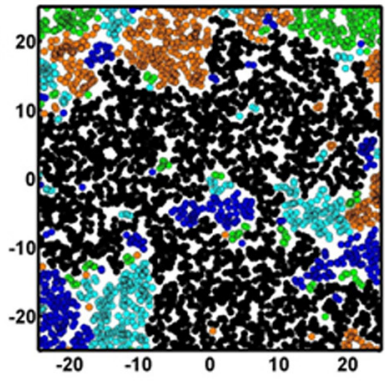

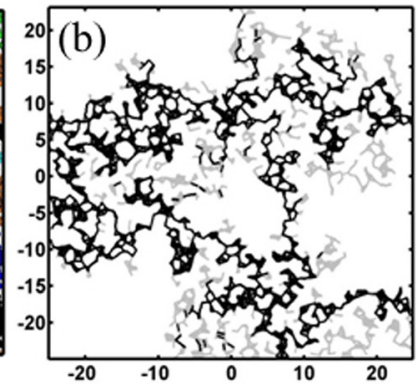

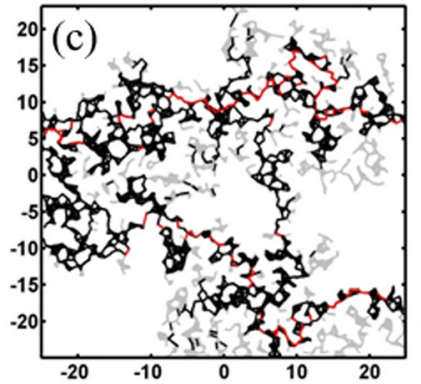

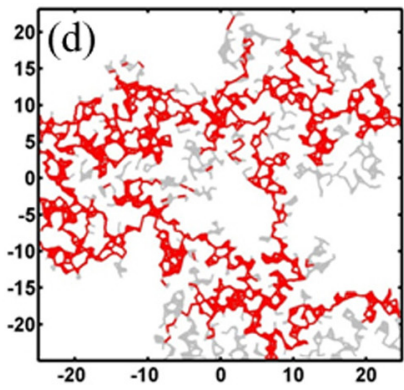

FIG. 1. Simulated percolating network of superconducting nanoparticles with coverage $p=0.69 .{ }^{37}$ (a) The "backbone" (or "spanning cluster") of nanoparticles that provides a current path between the contacts (left and right sides of the system) is shown in black, with other groups of connected particles shown in other colours. (b) Low applied currents $\left(I<I_{c}\right)$ : superconducting weak links in the backbone are shown in black while "dead ends" (that do not carry currents) are shown in grey. (c) $I=I_{c}$ : the critical weak links on the backbone have transitioned to the normal state (red). (d) $I \gg I_{c}$ : all connections are in the normal state, except for the dead ends. A similar behaviour is observed for all $p>p_{c}$, although as the coverage is increased the number of parallel current paths increases.

Here, we present a detailed analysis of transport data from percolating films of $25 \mathrm{~nm}$ superconducting $\mathrm{Pb}$ nanoparticles that demonstrates clearly that the "breakdown form" [Eq. (1)] is observed for samples with a very large range of normal state resistances, corresponding to different surface coverages, $p$, of the particles. A key aspect of this work is that we use pulsed current measurements to reveal percolation behaviour in low $R_{N}$ (high $p$ ) samples, where it is usually masked in DC measurements by the thermal effects. The critical exponent $a$ is found to be independent of both temperature and $p$. BKT behaviour [Eq. (2)] is observed for the low $R_{N}$ samples and is discussed elsewhere. ${ }^{22}$

The paper is organized as follows: Section II summarizes the aspects of percolation theory (and previous experimental studies) that are relevant to the present work and Sec. III describes the preparation of samples and measurement techniques; Section IV describes the experimental results, focusing first on the measured $V(I)$ curves, and then the determination of $I_{c}$ which finally shows that the data are well represented by Eq. (1). We conclude with a comparison of the measured power law exponent $a$ with values predicted or obtained elsewhere.

\section{PERCOLATION AND SUPERCONDUCTING FILMS}

Percolation theory describes the properties of systems composed of randomly occupied elements in $\mathrm{N}$-dimensions. ${ }^{47}$ In the present work, we are interested in nanoparticles randomly deposited on a flat surface with coverage $p$ : it is clear that a well-defined percolation threshold $p_{c}$ exists beyond which a connected network of particles spans the system, ${ }^{48}$ and that the system is well-described ${ }^{6,27,48-50}$ by a $2 \mathrm{D}$ continuum model, ${ }^{27,47,50,51}$ in which the particles are represented by overlapping discs. Continuum problems are often mapped onto lattice models with varying interaction strengths between $\operatorname{sites}^{27}$ and it is well established that the correlation length and conductivity $^{27,47,50,51}$ are governed by the power laws

$$
\xi_{p} \sim\left(p-p_{c}\right)^{-\nu},
$$

and

$$
G \sim\left(p-p_{c}\right)^{\mu}
$$

respectively, where $\nu=4 / 3$ and $\mu=1.3$ are universal values in $2 \mathrm{D}^{27,50}$ [Note that in the percolation literature, the conductivity (transport) exponent is usually written as $t$, but here we use $\mu$ because it is traditional to use $t=T / T_{c}$ for the reduced temperature in superconductivity].

For $p>p_{c}$, the spanning network (see Fig. 1) consists of a series of nodes, links, and blobs, ${ }^{52}$ where the mean distance between the nodes is given by the correlation length. ${ }^{53}$ When the particles are superconducting, the necks between the particles can be thought of as weak links, in which case, the critical current is also determined ${ }^{54}$ by $\xi_{p}$, and

$$
I_{c} \sim\left(p-p_{c}\right)^{m} .
$$

It is believed that the value of $m$ depends on the nature of the weak links: $m=\nu(d-1)=4 / 3$ for Josephson-coupling and $m=(\nu+1)(d-1) \sim 1.821$ for bulk-like depairing currents. ${ }^{6}$

\section{EXPERIMENTAL METHODS}

Lead nanoparticles with diameters of $25 \mathrm{~nm}$ were produced in a water-cooled inert gas aggregation source ${ }^{56}$ with a crucible temperature of $1050 \mathrm{~K}$, an Ar gas flow rate of $100-150 \mathrm{sccm}$, and a He gas flow of $0-30 \mathrm{sccm}$. The beam of nanoparticles was deposited under high vacuum onto a Si substrate with a $200 \mathrm{~nm} \mathrm{SiN} \mathrm{insulating} \mathrm{layer} \mathrm{supporting}$ $\mathrm{NiCr} / \mathrm{Au}$ electrodes separated by a $100 \mu \mathrm{m} \times 200 \mu \mathrm{m}$ gap. The current across the nanoparticle film (over a $100 \mu \mathrm{m}$ gap) was monitored during deposition and the temperature was controlled using a Janis ST-400 cryostat. The rate of nanoparticle deposition was monitored both before and after deposition through the use of a quartz crystal monitor and maintained at a rate of $0.2-0.4 \AA / \mathrm{s}$ (equivalent thickness). In order to prevent nanoparticle coalescence, the sample stage was maintained at a temperature of $10 \mathrm{~K}$ throughout the deposition. Here, we focus on samples with $R_{N} \sim 30$ $\Omega-100 \mathrm{k} \Omega\left(p>p_{c}\right)$. The measured transition temperatures are $T_{c} \sim 5.5-6.7 \mathrm{~K}$ (compared to $7.2 \mathrm{~K}$ in the bulk). The $V(I)$ curves were measured between 1.5 and $8 \mathrm{~K}$ using standard DC techniques and also using $55 \mu$ s pulses to avoid heating effects-see below.

During deposition, the conductance (Fig. 2) shows a clear transition from an exponential behavior below the percolation threshold ${ }^{49}$ to a power law behaviour of the 


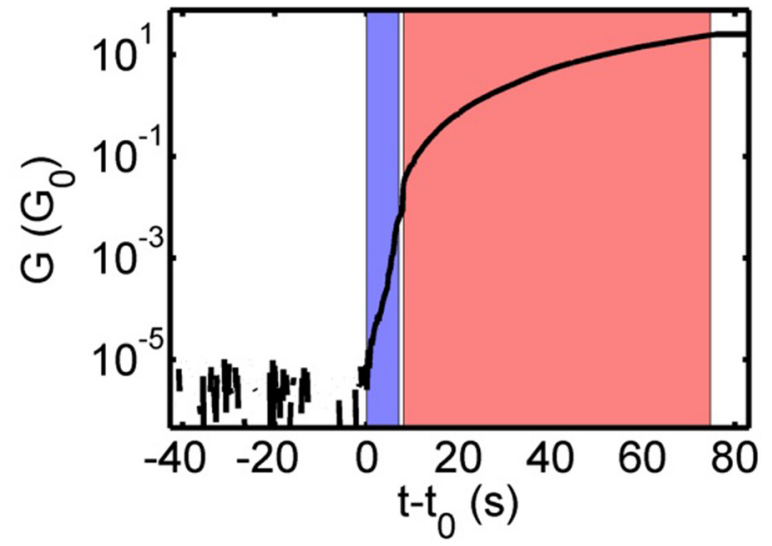

FIG. 2. Onset of conduction during deposition of nanoparticles showing the transition between exponential (blue) and power law (red) behavior; the percolation threshold occurs at the transition between the two regimes. ${ }^{49,55}$ The time axis is referenced to the first conductance increase above the noise floor. The deposition is halted at the end of the red region, after which the conductance remains constant.

form of Eq. (4) for $p>p_{c}$. This crossover typically occurs for $R_{N} \sim 150 \mathrm{k} \Omega$.

\section{RESULTS AND ANALYSIS}

\section{A. $\mathbf{R}(\mathrm{I})$ data}

$V(I)$ data were obtained for samples with a range of $R_{N}$ (corresponding to different nanoparticle coverages): an overview is best obtained when the resistance is plotted as a function of current as in Fig. 3. High coverage samples [low $R_{N}$, shown in Figs. 3(a) and 3(b)] act like thin homogenous films, ${ }^{57}$ with critical currents in the milliampere range and a large (up to several orders of magnitude) hysteretic jump to $R_{N}$ at $I^{*}$. The arrows indicate critical currents (see Sec. IV B), and $I^{*}$ indicates the current at which thermal effects drive a sudden transition to the normal state. ${ }^{57,58}$

$R(I)$ data for films with a lower density of nanoparticles, with $p$ close to but still above $p_{c}$, are shown in Figs. 3(c) and 3(d). These films are close to the percolation threshold, and so the critical current is determined by the weakest superconducting path in the system. ${ }^{34}$ The hysteresis is dramatically reduced compared to Figs. 3(a) and 3(b) because the thermal effects are much smaller.

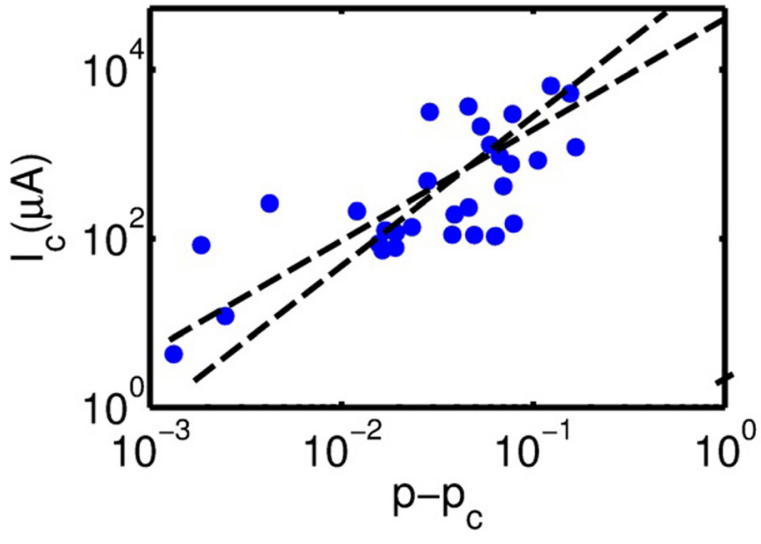

FIG. 4. Critical current vs $\left(p-p_{c}\right)$ for nanoparticle films. The expected power law for a $2 \mathrm{D}$ continuum system [Eq. (5)] ${ }^{6}$ is shown with exponents $m=4 / 3$ and $m=1.8$.

Below the percolation threshold [Fig. 3(e)], the $R(I)$ data change dramatically because tunnel gaps appear ${ }^{49}$ and there is no direct superconducting path across the system. In both the normal and superconducting state, samples show an activated behavior in both $R(T)$ and $R(I)$ measurements (decreasing resistance with increasing current or temperature). At lower coverages, still $\left(R_{N}>1 \mathrm{M} \Omega\right)$ a superconductor-to-insulator transition is observed. ${ }^{22}$

\section{B. Critical current}

In this section, we focus on measurements of the critical current, which are required for the detailed discussion of transport exponents in Sec. IV C. The critical current is defined by the point at which there is a distinct step in the measured resistance (in samples with low $R_{N}$ ) or by a kink in the $R(I)$ data, as shown in Fig. 3. It is important to note that in the case of high coverage samples $\left(p \gg p_{c}\right), I^{*}$ occurs prior to the true critical current.

The coverage dependence of the critical current is shown in Fig. 4. The data are compared with the expected power law [Eq. (5)] for a continuum system of overlapping discs with Josephson coupling between particles $(m=4 / 3)$ and with bulk-like de-pairing currents $(m=1.8) .{ }^{6}$ The scatter in the data are too great to clearly distinguish between the two cases, but since the necks between particles in our
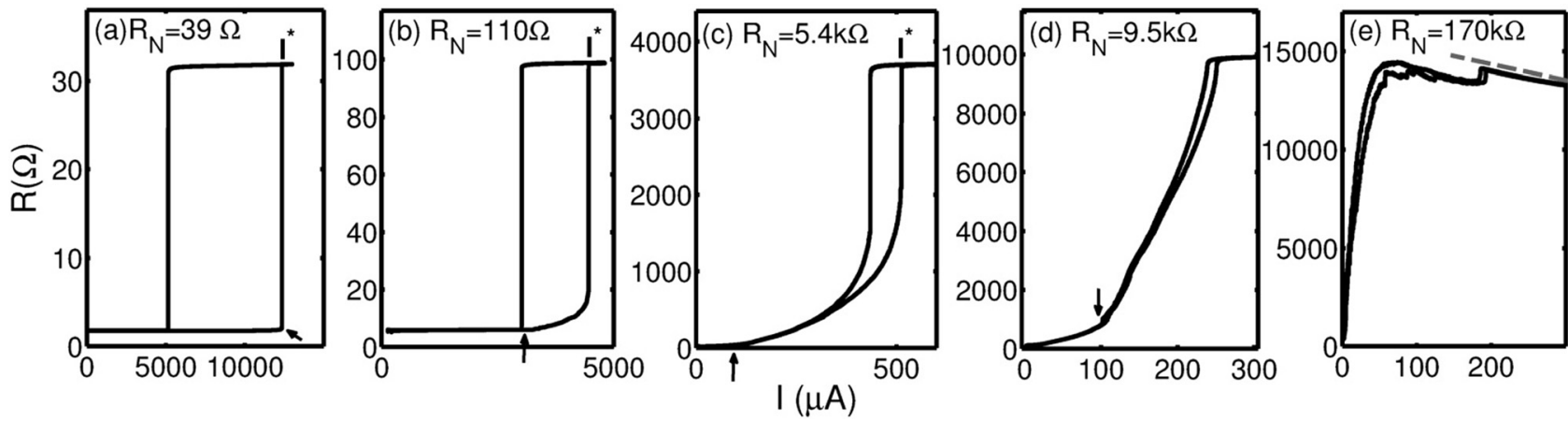

FIG. 3. Low temperature ( $\sim 2 \mathrm{~K}) R(I)$ data for selected samples with $R_{N}=39,110,5400,9500$, and $170000 \Omega$. The percolation threshold is between $\mathrm{d}$ and e. The critical currents are indicated (arrows), as well as a thermal instability current $I^{*}$. The dotted line in curve (e) indicates the increase in resistance at lower current; a resistance drop is observed at low currents (in contrast to genuinely insulating samples-not shown-which are highly resistive at low currents and low temperatures). 


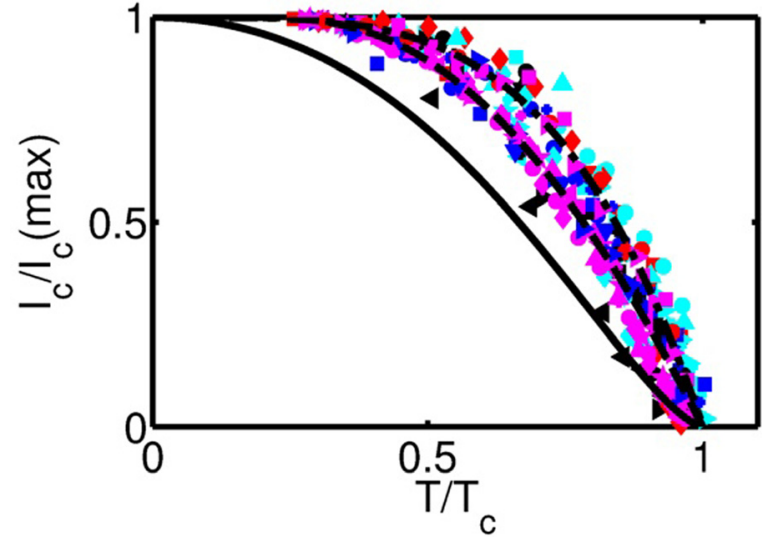

FIG. 5. $I_{c}$ vs t. Reference curves shown for $1-t^{4}$ (dashed-dotted), Ambegaokar-Baratoff tunnel junction JJ form $I_{c} R_{N}=(\pi \Delta / 2 e) \tan h(\Delta / 2 k T)$ (dash), and thin films $\left(1-t^{2}\right)^{3 / 2}\left(1+t^{2}\right)^{1 / 2}$ (solid). The color scale identifies the normal state resistance in $\Omega$ of the 32 samples shown: red $R_{N}<100$, magenta $100<R_{N}<1000$, cyan $1000<R_{N}<5000$, black $5000<R_{N}$ $<10000$, and blue $10000>R_{N}$.

samples are smaller than the superconducting coherence length $\xi_{s}$ (not to be confused with the percolation correlation length $\xi_{p}$ ), it seems likely that it is the Josephson limit that is relevant.

The critical current at $p_{c}$ should be on the order of that for a single nanoparticle, which for a $30 \mathrm{~nm}$ diameter wire and $J_{c}=10^{-7} \mathrm{~A} / \mathrm{cm}^{2}$ as measured in $\mathrm{Pb}$ thin films, ${ }^{59}$ gives an estimate of the critical current $J_{c} \pi r^{2}=72 \mu \mathrm{A}$. This estimate is an upper limit (as the neck size between uncoalesced particles can be considerably smaller), but is consistent with the data in Fig. 4. For $p \gg p_{c}$, there are many parallel paths ( $N=3300-6600$ for a $100-200 \mu \mathrm{m}$ film with $30 \mathrm{~nm}$ clusters $)$ and this suggests $I_{c} \sim N * 72 \mu \mathrm{A} \sim 240-480 \mathrm{~mA}$, which is an order of magnitude higher than that observed in the thickest films. The lower observed critical current in these films is consistent with our expectation that even at high coverages, disorder is significant.

The temperature dependence of the critical current is shown in Fig. 5 for a range of coverages: the observed
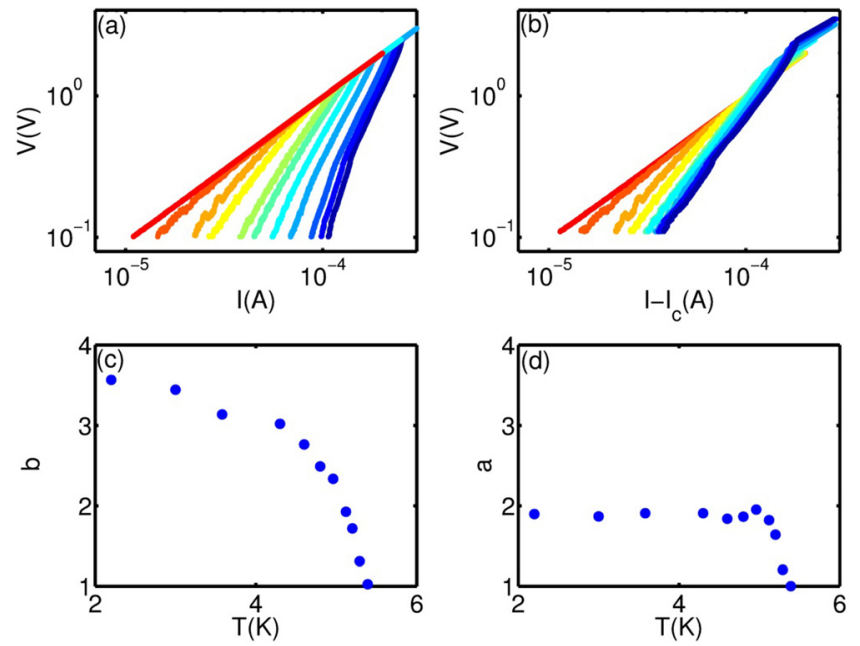

FIG. 6. V(I) data for a sample with $R_{N}=9.5 \mathrm{k} \Omega,(p \sim 0.678)$. (a) Raw $V(I)$ data $[T$ (right to left), 2.20-5.39K], (b) breakdown plot [Eq. (1)], (c) fitted exponents $b$ from Eq. (2) of the data in (a), and (d) fitted exponents $a$ for the data in (b). functional form is remarkably similar for all samples. The decrease in $I_{c}$ with increasing $T$ lies between the Ambegaokar-Baratoff ${ }^{60}$ form for tunnel coupled Josephson junctions and an empirical $1-t^{4}$ form (where $t=T / T_{c}$ ), which has been previously reported in granular films. ${ }^{61,62}$

Apart from this, we note that a crossover to the Ginzburg-Landau thin film dependence $\left((1-t)^{3 / 2}\right.$ close to $\left.T_{c}, 0.9<t<0.95\right)$ is observed in some low resistance samples (red and magenta samples in Fig. 5). $\mathrm{Clem}^{63}$ argued that the crossover in granular films is a result of a current induced gap suppression when the Josephson energy is comparable to the condensation energy per grain $\left[\sim 2 \xi_{s}(T) / a_{0}\right.$, where $a_{0}$ is the effective grain size and $\xi_{s}(0) \sim 80 \mathrm{~nm}$ (Ref. 59)].

\section{Transport exponents}

The main focus of this work is to understand the effects of percolation on the $V(I)$ curves and examine the range of validity of Eq. (1). The $V(I)$ curves shown in Fig. 6(a) are typical of low coverage samples $\left(p_{c}<p \leqq 0.73, R_{N} \gtrsim 5 \mathrm{k} \Omega\right)$, with a noticeable curvature and weakly temperature dependent exponents $b \sim 2-5$ [Fig. 6(c)]. When the data are replotted as in Fig. 6(b), it becomes clear that the data are consistent with the breakdown form [Eq. (1)], where the exponent $a$ is temperature independent [Fig. 6(d)] except near $T_{c}$.

The same procedure yields similar exponents $a$ for samples with a wide range of coverages, as discussed below, but we will first consider the arguments against the alternative explanation of the data in terms of the power law Eq. (2) which would suggest, for example, BKT behaviour. First, as noted in the previous paragraph, plots such as Fig. 6(a) are consistently non-linear on a log-log scale for all samples. Second, the expected linear temperature dependence ${ }^{64}$ of $b$ below $T_{B K T}$ is not observed in Fig. 6(c), and $b$ converges to 1 near the transition temperature $\left(T_{c}\right.$ or $T_{B K T}$ depending on the interpretation) rather than the expected value $b=3$. Finally, we do in fact observe BKT behaviour in high coverage (small $R_{N}$ ) samples at lower currents - see for example the data in Fig. 8(a) at voltages less than $\sim 10^{-4} \mathrm{~V}$. The BKT transition in these samples is, however, complex ${ }^{22}$ and here we simply note that vortex unbinding is observed for $I<I_{c}$, whilst percolative behaviour in the form of Eq. (1) is associated with the regime $I>I_{c}$.

For samples with $p \gtrsim 0.7\left(R_{N} \lesssim 1000 \Omega\right)$, there is a sharp transition to the normal state at $I^{*}$ and the behaviour of the breakdown form is not immediately obvious in measurements with DC currents (blue curve in Fig. 7). However pulsed current measurements allow $V(I)$ data to be measured well beyond $I^{*}$ (up to an order of magnitude higher, though with a higher noise threshold-black curve in Fig. 7).

Figure 8 presents data for a sample with a smaller $R_{N}$ and shows that the DC (a) and pulsed (b) measurements are consistent for a wide range of temperatures: Fig. 8(c) shows that the DC and pulsed data overlap nicely. Figure 8(d) shows that the pulsed current data fit the breakdown form [Eq. (1)] with $a=2.1-2.3$ - these fitted exponents agree well with the fits of DC measurements taken for samples close to the percolation threshold (Fig. 9). 


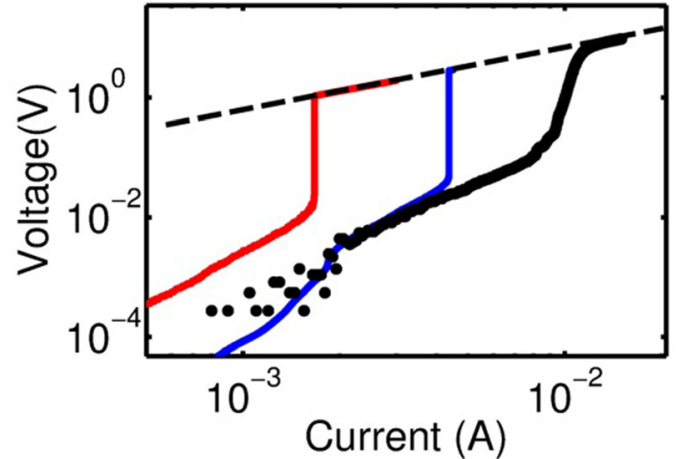

FIG. 7. Comparison of pulsed (black, $1.70 \mathrm{~K}$ ) vs DC current measurements (blue and red $1.70 \mathrm{~K}$ and $5.51 \mathrm{~K}$, respectively), for a sample with $R_{N}=645$ $\Omega$. In DC measurements, the jump to the normal state occurs at $I^{*}$ but pulsed measurements allow the $V(I)$ data to be obtained for $I>I^{*}$. The dashed line indicates $R_{N}$.

In practice, the correct choice of $I_{c}$ means that the $V(I)$ data at all temperatures yield a single temperatureindependent slope on plots of $V$ versus $I-I_{c}$, whereas plots of this form for incorrect choices of $I_{c}$ yield either a strongly temperature dependent $a$, or significant curvature. Our fitting procedure is validated by fits using Eq. (1) for samples with a wide range of coverages. Figure 9 shows that the fitted exponent $a$ is both temperature independent and coverage independent, with $a=2.1 \pm 0.2$ for all $p>p_{c}{ }^{65}$ The fitted exponents are remarkably consistent over a wide range of coverages, apart from 3 points close to $p_{c}$ where large fluctuations are expected.

\section{DISCUSSION}

The power law in the breakdown form [Eq. (1)] has been predicted in several different ways, ${ }^{34-36,66}$ but, as reviewed in this section, it is not clear in the previous literature what the value of the exponent $a$ is expected to be, or in what range of $p$ and $I$ Eq. (1) is valid. Prester ${ }^{36}$ showed that a linear expansion of an effective (current-dependent) coverage about $I_{c}$ together with Eq. (4) leads to $V \propto\left(I-I_{c}\right)^{\mu+1}$ and hence $a=2.3$ in 2 D. Granato ${ }^{35}$ used scaling calculations by Lee ${ }^{67}$ to derive $a=1+\mu /(\nu(d-1))=1.98$ in $2 \mathrm{D}$ at (or close to) $p_{c}$. For higher coverages, the same authors used dimensional arguments and finite size scaling to show that $a=(z+1) \nu_{1}$,

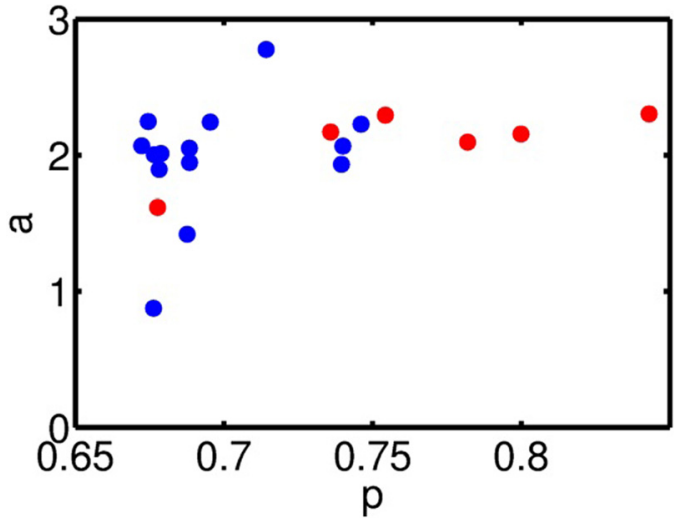

FIG. 9. Low temperature $(T=2 \mathrm{~K})$ fitted exponents $a$ as a function of $p$ obtained from plots of the form of Fig. 6(b) for samples with a wide range of values of $R_{N}$. The fits for samples with $R_{N}<1000 \Omega$ ( $p \geq 0.73$, red points) are taken from pulsed measurements to avoid the heating effects.

where $z=0.9-1.2$ and $\nu_{1}=1-1.1$, leading to $a \sim 1.9-2.4$, in reasonable agreement with their JJ array simulations $(a=2.5 \pm 0.2)$, and suggested that this value may be universal. Bradley ${ }^{34}$ estimated $a=2.0 \pm 0.1$ from both finite size scaling of lattice simulations at $p_{c}$ and from a nodeslinks approximation for the overlapping disc problem, as either $I_{c}$ and $p_{c}$ are approached from above. Roux ${ }^{66}$ simulated a $2 \mathrm{D}$ network of voltage activated resistors that is complementary to the superconducting system of interest here. They proposed a mean field argument to show that a distribution of voltage thresholds should lead directly to $I \propto$ $\left(V-V_{g}\right)^{2}$ over the entire range of V. It is clear that a similar argument applies to the present superconducting system with a distribution of critical currents, ${ }^{68}$ leading to Eq. (1) with $a=2$.

The literature includes several previous experimental observations of power laws of the breakdown form, in studies of HTSCs, ${ }^{28,69-73}$ as well as in granular systems modeled as Josephson junction arrays. ${ }^{74}$ In HTSCs, the range of observed exponents $a=2-3$ is quite large, though the reasons for this are not clear. The uncertainties in the literature are further compounded by measurements of disordered Ta grains in a $3 \mathrm{D}$ matrix, ${ }^{74}$ where it was found that $a=2.1$ \pm 0.2 : in $3 \mathrm{D}$ one should expect a larger value of $a$ than in $2 \mathrm{D}$.
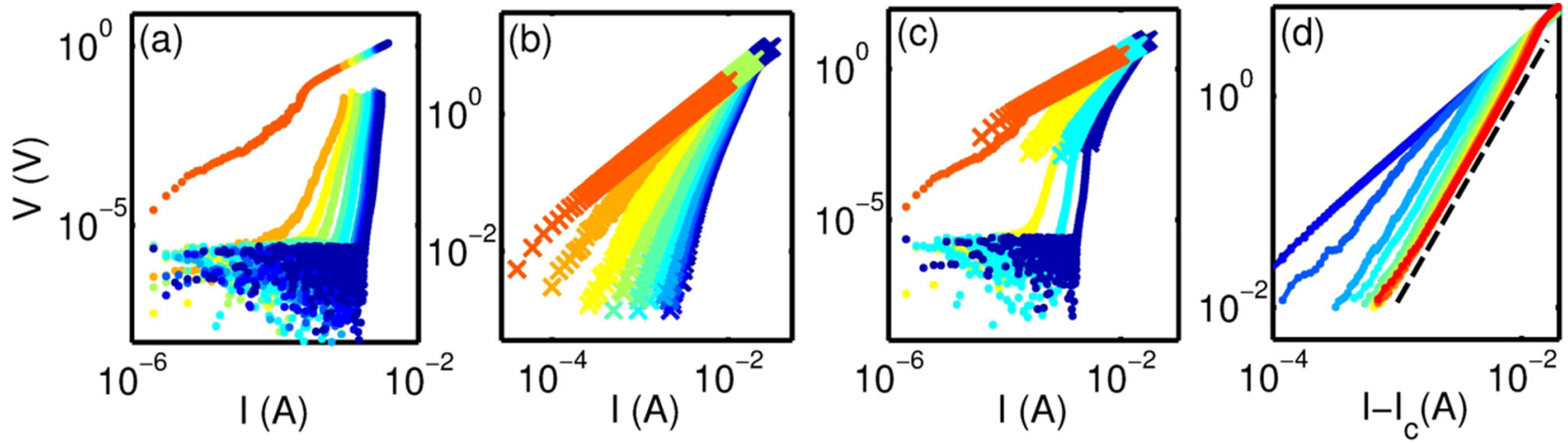

FIG. 8. DC and pulsed measurements on the same sample $\left(R_{N}=300 \Omega\right)[T=1.80,2.70,3.06,3.40,4.00,4.30,4.50,4.70,4.80,5.06$, and $5.51 \mathrm{~K}$ (right to left)]. (a) Raw $V(I)$ data, (b) pulsed data for $55 \mu$ s pulses, (c) combined pulsed and DC measurements (selected temperatures), pulsed data at higher currents (crosses), and the noise floor at low currents is shown, and (d) pulsed data after $I_{c}$ subtraction with the linear region indicated $(a=2.2)$. 
Our main results (see Sec. IV) are that Eq. (1) applies over a range of currents that is typically $1<I / I_{c} \lesssim 10$, and that the power law exponent $a=2.1 \pm 0.2$ is constant for all samples with coverages in the range of $p_{c}<p<0.85$ $\left(100 \mathrm{k} \Omega>R_{N}>30 \Omega\right)$. These results are broadly consistent with previous experiments and the theoretical results and simulations of Refs. 28 and 33-35. However, it remains unclear why the values of $a$ are so consistent over such a wide range of coverages since in every case, the models in the existing literature are strictly valid over a much narrower range. For example, Ref. 34 focuses on a continuum system, and at the first sight, its results are precisely consistent with our experimental results. However, the results in Ref. 34 are valid only for $p=p_{c}$ or for a nodes-links model, and the authors themselves point out that the internal structure of the "blobs" should be considered. Similarly, Ref. 66 at the first sight appears to demonstrate on very general grounds that $a$ should always be precisely 2-but this is clearly not consistent with the other literature cited above which finds $a \neq 2$. It seems therefore that new and detailed simulations are required to fully resolve the range of validity of Eq. (1). Such simulations would need to consider the broad range of coverages of our experiments and include the self-similar internal structure of the blobs in continuum percolation, ${ }^{53}$ and also to establish finally whether the form of Eq. (1) is expected over a broad range of applied currents ${ }^{66}$ or only as $I_{c}$ is approached from above. ${ }^{34}$

\section{CONCLUSIONS}

We have fabricated thin percolating films of $25 \mathrm{~nm}$ superconducting $\mathrm{Pb}$ nanoparticles with a wide range of surface coverages corresponding to normal state resistances between $20 \Omega$ and $50 \mathrm{M} \Omega$ and have focussed here on samples with $R_{N}<100 \mathrm{k} \Omega$ that exhibit superconducting behaviour. The $V(I)$ characteristics of these films exhibit 3 regimes of behaviour: (1) high coverage samples $\left(p \gg p_{c}, R_{N}<1 \mathrm{k} \Omega\right.$ ) exhibit no measurable voltage above the noise floor until a sharp jump to the normal state at a high DC current $\left(I^{*}\right)$ : pulsed current measurements, however, allow a behaviour that is well described by Eq. (1) to be revealed for a range of currents that far exceeds $I^{*}$; (2) samples with $1 \mathrm{k} \Omega<R_{N}$ $<100 \mathrm{k} \Omega$ (i.e., near to $p_{c}$ ), exhibit nearly linear $V(I)$ curves at low currents and then a transition to the breakdown behaviour [Eq. (1)] at $I_{c}$; and (3) high resistance samples $\left(p<p_{c}\right.$, $R_{N}>100 \mathrm{k} \Omega$ ) show an activated type resistance associated with an insulating state. We emphasize that Eq. (1) is observed for $I>I_{c}$, and is distinct from the vortex unbinding/ BKT physics that typically gives rise to Eq. (2).

Our measurements provide direct experimental evidence of the near universal value of the breakdown power law with an exponent $a=2.1 \pm 0.2$ over a wide range of coverages, applied currents, and temperatures. This result suggests that the break-down form [Eq. (1)] is more generally applicable than would be expected from the existing theory: detailed simulations or more in-depth theoretical analysis are required. Such an analysis would be of significant value because $V(I)$ measurements have the potential to add significantly to our understanding of a number of percolating/ superconducting systems, and could provide an important probe of the superconductor-insulator transition in thin films and granular systems, in addition to the continuum percolation systems investigated here.

\section{ACKNOWLEDGMENTS}

The authors gratefully acknowledge financial support from the MacDiarmid Institute, the Marsden Fund, the Fund for Scientific Research - Flanders (FWO), and the KU Leuven Concerted Research Action GOA/14/007.

${ }^{1}$ A. Davidson and M. Tinkham, Phys. Rev. B 13, 3261 (1976).

${ }^{2}$ G. Deutscher and M. Rappaport, J. Phys. Lett. 40, 219 (1979).

${ }^{3}$ G. Deutscher, O. Entin-Wohlman, S. Fishman, and Y. Shapira, Phys. Rev. B 21, 5041 (1980).

${ }^{4}$ S. Kirkpatrick, AIP Conf. Proc. 58, 79 (1980).

${ }^{5} \mathrm{P}$. de Gennes, in Percolation, Localization, and Superconductivity, edited by A. M. Goldman and S. A. Wolf (Springer US, New York, 1984), pp. 83-94.

${ }^{6}$ M. Octavio, A. Octavio, J. Aponte, R. Medina, and C. Lobb, Phys. Rev. B 37, 9292 (1988).

${ }^{7}$ Y. Strelniker, A. Frydman, and S. Havlin, Phys. Rev. B 76, 224528 (2007).

${ }^{8}$ M. Eisterer, M. Zehetmayer, and H. Weber, Phys. Rev. Lett. 90, 247002 (2003).

${ }^{9}$ D. Haviland, Y. Liu, and A. Goldman, Phys. Rev. Lett. 62, 2180 (1989).

${ }^{10}$ H. Jaeger, D. Haviland, B. Orr, and A. Goldman, Phys. Rev. B 40, 182 (1989).

${ }^{11}$ M. Choi, J. Chung, and D. Stroud, Phys. Rev. B 35, 1669 (1987).

${ }^{12}$ M. Forrester, H. Lee, M. Tinkham, and C. Lobb, Phys. Rev. B 37, 5966 (1988).

${ }^{13}$ D. Harris, S. Herbert, D. Stroud, and J. Garland, Phys. Rev. Lett. 67, 3606 (1991).

${ }^{14}$ M. Fisher, Phys. Rev. B 36, 1917 (1987).

${ }^{15}$ D. Haviland, H. Jaeger, B. Orr, and A. Goldman, Phys. Rev. B 40, 719 (1989).

${ }^{16}$ L. Merchant, J. Ostrick, R. Barber, and R. Dynes, "Crossover from phase fluctuation to amplitude-dominated superconductivity: A model system," Phys. Rev. B 63, 134508 (2001).

${ }^{17}$ Y. Dubi, Y. Meir, and Y. Avishai, Nature 449, 876 (2007).

${ }^{18}$ A. Erez and Y. Meir, Phys. Rev. Lett. 111, 187002 (2013).

${ }^{19}$ M. Ovadia, D. Kalok, B. Sacépé, and D. Shahar, Nat. Phys. 9, 415 (2013).

${ }^{20}$ R. Koushik, S. Kumar, K. R. Amin, M. Mondal, J. Jesudasan, A. Bid, P. Raychaudhuri, and A. Ghosh, Phys. Rev. Lett. 111, 197001 (2013).

${ }^{21}$ Z. Han, A. Allain, and H. Arjmandi-Tash, Nat. Phys. 10, 380 (2014).

${ }^{22}$ A. Nande, S. Fostner, J. Grigg, A. Smith, K. Temst, M. J. Van Bael, and S. A. Brown, Nanotechnology 28, 165704 (2017).

${ }^{23}$ S. Bose, P. Raychaudhuri, R. Banerjee, P. Vasa, and P. Ayyub, Phys. Rev. Lett. 95, 147003 (2005).

${ }^{24}$ B. Sacépé, C. Chapelier, T. Baturina, V. Vinokur, M. Baklanov, and M. Sanquer, Phys. Rev. Lett. 101, 157006 (2008).

${ }^{25}$ M. Mondal, A. Kamlapure, M. Chand, G. Saraswat, S. Kumar, J. Jesudasan, L. Benfatto, V. Tripathi, and P. Raychaudhuri, Phys. Rev. Lett. 106, 047001 (2011).

${ }^{26}$ A. Allain, Z. Han, and V. Bouchiat, Nat. Mater. 11, 590 (2012).

${ }^{27}$ S. Feng, B. Halperin, and P. Sen, Phys. Rev. B 35, 197 (1987).

${ }^{28}$ B. Zeimetz, B. Glowacki, and J. Evetts, Eur. Phys. J. B 29, 359 (2002).

${ }^{29}$ R. Fazio and H. van, der Zant, Phys. Rep. 355, 235 (2001).

${ }^{30}$ Y.-J. Yun, I.-C. Baek, and M.-Y. Choi, Phys. Rev. Lett. 97, 215701 (2006).

${ }^{31}$ L.-H. Tang and Q.-H. Chen, Phys. Rev. B 67, 024508 (2003).

${ }^{32}$ S. John and T. Lubensky, Phys. Rev. B 34, 4815 (1986).

${ }^{33}$ M. Prester, Supercond. Sci. Technol. 11, 333 (1998).

${ }^{34}$ R. Bradley, D. Kung, P. Strenski, and S. Doniach, Phys. B 152, 282 (1988).

${ }^{35}$ E. Granato and D. Domínguez, Phys. Rev. B 56, 14671 (1997).

${ }^{36}$ M. Prester, Phys. Rev. B 54, 606 (1996).

${ }^{37}$ A. Smith, "Simulating percolating superconductors," Ph.D. thesis (University of Canterbury, 2014).

${ }^{38}$ J. Fontcuberta and J. Jurado, Z. Phys. B-Condens. Matter 87, 21 (1992). 
${ }^{39}$ G. Blatter, M. Feigel'man, and V. Geshkenbein, Rev. Mod. Phys. 66, 1125 (1994).

${ }^{40}$ V. Berezinskii, Sov. J. Exp. Theor. Phys. 34, 610 (1972).

${ }^{41}$ J. Kosterlitz and D. Thouless, J. Phys. C: Solid State Phys. 6, 1181 (1973).

${ }^{42}$ Y. M. Blanter, Y. E. Lozovik, and A. Y. Morozov, Phys. Scr. 52, 237 (1995).

${ }^{43}$ B. Costa, P. Coura, and S. Leonel, Phys. Lett. A 377, 1239 (2013).

${ }^{44}$ L. Benfatto, C. Castellani, and T. Giamarchi, Phys. Rev. B 80, 214506 (2009).

${ }^{45}$ S. Herbert, Y. Jun, R. Newrock, and C. Lobb, Phys. Rev. B 57, 1154 (1998).

${ }^{46}$ M. V. Simkin and J. M. Kosterlitz, Phys. Rev. B 55, 11646 (1997).

${ }^{47}$ D. Stauffer and A. Aharony, Introduction to Percolation Theory, 2nd ed. (CRC Press, New York, 1994).

${ }^{48}$ J. Schmelzer, S. A. Brown, A. Wurl, M. Hyslop, and R. J. Blaikie, Phys. Rev. Lett. 88, 226802 (2002).

${ }^{49}$ S. Fostner, R. Brown, J. Carr, and S. A. Brown, Phys. Rev. B 89, 075402 (2014).

${ }^{50}$ E. T. Gawlinski and H. E. Stanley, J. Phys. A: Math. Gen. 14, L291 (1981).

${ }^{51}$ J. Quintanilla and S. Torquato, Phys. Rev. E 54, 4027 (1996).

${ }^{52}$ H. Stanley, J. Phys. A: Math. Gen. 10, L211 (1977).

${ }^{53}$ A. Coniglio, J. Phys. A: Math. Gen. 3829, 3829 (1982).

${ }^{54}$ G. Deutscher and O. Entin-Wohlman, AIP Conf. Proc. 58, 23 (1980).

${ }^{55}$ G. Ambrosetti, I. Balberg, and C. Grimaldi, Phys. Rev. B 82, 134201 (2010).

${ }^{56}$ R. Reichel, J. G. Partridge, A. D. Dunbar, S. A. Brown, O. Caughley, and A. Ayesh, J. Nanopart. Res. 8, 405 (2006).

${ }^{57}$ J. Bremer and V. Newhouse, Phys. Rev. 116, 309 (1959).
${ }^{58}$ J. Maza, G. Ferro, J. Veira, and F. Vidal, Phys. Rev. B 78, 094512 (2008).

${ }^{59}$ J. Cuppens, C. P. Romero, P. Lievens, and M. J. Van Bael, Phys. Rev. B 81, 064517 (2010).

${ }^{60}$ V. Ambegaokar and A. Baratoff, Phys. Rev. Lett. 11, 104 (1963).

${ }^{61}$ K. Das Gupta, S. Soman, G. Sambandamurthy, and N. Chandrasekhar, Phys. Rev. B 66, 144512 (2002).

${ }^{62}$ Note that because it is not possible to measure $I_{c}(T=0)$, there is a small uncertainty in the correct normalisation of the data: to manage this, we scale the value of $I_{c}$ at the minimum measurement temperature to the empirical $1-t^{4}$ curve. Alternative scaling procedures produce similar results.

${ }^{63}$ J. Clem, B. Bumble, S. Raider, W. Gallagher, and Y. Shih, Phys. Rev. B 35, 6637 (1987)

${ }^{64}$ A. Kadin, K. Epstein, and A. Goldman, Phys. Rev. B 27, 6691 (1983).

${ }^{65}$ This uncertainty calculated from the standard deviation of the fitted exponents for both pulsed and DC measurements together.

${ }^{66}$ S. Roux and H. J. Herrmann, Europhys. Lett. 4, 1227 (1987).

${ }^{67}$ K. Lee and D. Stroud, Phys. Rev. B 45, 2417 (1992).

${ }^{68}$ E. Guyon, S. Roux, and A. Hansen, Rep. Prog. Phys. 53, 373 (1990).

${ }^{69}$ S. Bungre and S. Cassidy, Supercond. Sci. Technol. 4, S250 (1991).

${ }^{70}$ P. Peyral, C. Lebeau, J. Rosenblatt, A. Raboutou, C. Perrin, O. Peña, and M. Sergent, J. Less Common Met. 151, 49 (1989).

${ }^{71}$ S. Bungre, R. Meisels, A. Caplin, and S. Male, Phys. C: Supercond. 162-164, 1171 (1989).

${ }^{72}$ E. Babić, M. Prester, D. Babić, P. Nozar, P. Šastný, and F. Matacotta, Solid State Commun. 80, 855 (1991).

${ }^{73}$ P. England, T. Venkatesan, X. Wu, and A. Inam, Phys. Rev. B 38, 7125 (1988).

${ }^{74}$ C. Lebeau and J. Rosenblatt, Europhys. Lett. 1, 313 (1986). 